SUINAGA, F.A.; CASALI, V.W.D.; PICANÇO, M.C.; SILVA, D.J.H. Capacidade combinatória de sete caracteres de resistência de Lycopersicon spp. à traça do tomateiro. Horticultura Brasileira, Brasília, v.22, n.2, p.243-248, abril-junho 2004.

\title{
Capacidade combinatória de sete caracteres de resistência de Lycopersicon spp. à traça do tomateiro
}

\author{
Fábio A. Suinaga ${ }^{1}$; Vicente W.D. Casali ${ }^{2}$; Marcelo C. Picanço ${ }^{3}$; Derly J.H. da Silva ${ }^{2}$ \\ ${ }^{1}$ Embrapa Algodão, R. São Paulo, 790, 78850-000 Primavera do Leste-MT; ${ }^{2}$ UFV, Depto. Fitotecnia; ${ }^{3}$ UFV, Depto. Biologia Animal, \\ 36571-000 Viçosa-MG; E-mail: suinaga@cnpa.embrapa.br
}

\section{RESUMO}

Com o objetivo de estudar as capacidades específica e geral de combinação (CEC e CGC, respectivamente) entre dois grupos de Lycopersicon spp., obtiveram-se híbridos interespecíficos de tomateiro utilizando-se o modelo de cruzamento dialélico parcial. Foram avaliadas sete características de resistência destes grupos ao ataque de Tuta absoluta. Foram observadas diferenças significativas quanto a maioria das características com relação às capacidades geral e específica de combinação. Baseado na CGC, os genótipos Ângela, Santa Clara, LA 716, e LA 1777 são os parentais mais indicados a serem incluídos em programas de melhoramento do tomateiro que visem aumentar a resistência a T. absoluta. As combinações híbridas com maiores valores conjuntos de CEC e heterose foram Ângela x PI 126445, Ângela x PI 127826, IPA-5 x LA 1777, e Santa Clara x LA 716 .

Palavras-chave: Tuta absoluta, tomate, melhoramento de plantas, biometria.

\begin{abstract}
Combining ability of seven resistance characteristics of Lycopersicon spp. to tomato leafminer

Using the partial diallel methodology, interspecific hybrids of tomato were obtained, in order to study the general and specific combining abilities (GCA and SCA, respectively) between two groups of Lycopersicon spp. Seven characteristics of tomato resistance to Tuta absoluta were evaluated. Significant differences regarding the majority of the evaluated traits were observed. Based on GCA, the genotypes Ângela, Santa Clara, LA 716 and LA 1777 are the most indicated to be used in a breeding program to improve the tomato resistance to T. absoluta. The best hybrids, considering SCA and heterosis, were Ângela x PI 126445, Ângela x PI 127826, IPA-5 x LA 1777 and Santa Clara x LA 716.
\end{abstract}

Keywords: Tuta absoluta, tomato, plant breeding, biometry.

(Recebido para publicação em 24 de março de 2003 e aceito em 20 de março de 2004)

traça do tomateiro, Tuta absoluta
(Meyrick) (Lepidoptera: Gelechiidae), foi constatada pela primeira vez no Brasil em 1980 estando hoje dentre as principais pragas da tomaticultura nacional (Guedes et al., 1995; Picanço et al., 1997).

O controle desta praga é feito principalmente com inseticidas, sendo realizadas em casos extremos, até três pulverizações semanais. Como conseqüência do uso indiscriminado de inseticidas tem-se verificado a eliminação das populações de inimigos naturais (Melo e Campos, 2000), contaminação do meio ambiente (Moreira e Oliveira, 1997), e o desenvolvimento de resistência da praga ao inseticida (Siqueira et al., $2000 \mathrm{e}$ 2001). Neste panorama, dentre as principais formas de reduzir a dependência dos pesticidas na agricultura está o desenvolvimento de plantas resistentes a insetos. Assim, a resistência de plantas é uma importante ferramenta no manejo integrado de pragas, pois constitui-se tática efetiva, econômica e sustentável (Pedigo, 2002).
Desta forma, diversas espécies silvestres de tomateiro têm sido identificadas como fontes de resistência a insetos, destacando-se Lycopersicon hirsutum f. glabratum (PI 134417) expressando resistência a Leptinotarsa decemlineata (Coleoptera: Chrysomelidae), Spodoptera exigua (Lepidoptera: Noctuidae), Bemisia argentifolii (Homoptera: Aleyrodidae), Myzus persicae (Homoptera: Aphididae) e Keiferia lycopersicella (Lepidoptera: Gelechiidae) (Farrar e Kennedy, 1991; Leite et al., 1999 a,b; Lin e Trumble, 1986). Os acessos PI 127826 e PI 127827 de L. hirsutum f. hirsutum tem resistência a T. absoluta e B. argentifolii (Campos et al., 1999; Toscano e Boiça Jr., 1999). Além destes, L. hirsutum f typicum (LA 1777, LA 2329 e PI 126445) possuem resistência a L. decemlineata, S. exigua, T. absoluta (Ecole et al., 1999; Eigenbrode e Trumble, 1993, 1994; Eigenbrode et al. 1994, 1996). A introdução LA 716 de $L$. pennellii, apresentou resistência a Liriomyza trifolii (Diptera:
Agromyzidae), Macrosiphum euphorbiae (Homoptera: Aphididae) e B. argentifolii (Hawthorne et al., 1992; Goffreda et al, 1989; Campos et al., 1999).

Apesar da detecção de fontes promissoras de resistência à traça-do-tomateiro, nos programas de melhoramento que visam incorporar tais características agronômicas, perde-se o nível adequado de resistência à traça do tomateiro. As causas de tal fato podem ser atribuídas a diversos fatores como dificuldade na transferência das características de resistência às cultivares comerciais suscetíveis e o desconhecimento da genética da resistência (Farrar e Kennedy 1987, 1991).

Segundo Ramalho et al. (1993) e Amaral Júnior et al. (1996), a melhor alternativa nesta situação, seria o desenvolvimento da hibridação visando a recombinação da variabilidade propiciando cultivares adaptadas às novas demandas. Portanto, torna-se indispensável o estudo do comportamento per se dos genitores e das combinações híbri- 
Tabela 1. Estimativas dos efeitos da capacidade geral ( $\hat{\mathrm{g}}$ ) de combinação para sete caracteres de resistência de tomateiros a T. absoluta. Viçosa, UFV, 2001.

\begin{tabular}{|c|c|c|c|c|c|c|c|}
\hline \multirow{2}{*}{ Genótipos } & \multicolumn{7}{|c|}{ Caracteres $1 /$ Efeitos gi } \\
\hline & MNP & MNG & MLV & PLV & PES & MPU & PPU \\
\hline Ângela & 0,74 & 1,27 & $-4,88$ & 2,42 & 0,14 & 3,22 & 0,42 \\
\hline IPA-5 & 3,03 & 1,33 & 2,15 & $-1,82$ & $-0,23$ & 1,02 & $-0,50$ \\
\hline Santa Clara & $-2,93$ & $-1,49$ & 4,79 & $-2,62$ & 0,00 & $-0,13$ & $-0,55$ \\
\hline TX 401-08 & $-0,84$ & $-1,12$ & $-2,06$ & 2,01 & 0,09 & $-4,10$ & 0,64 \\
\hline LA 716 & $-4,38$ & $-2,36$ & 8,33 & $-3,49$ & $-0,21$ & 7,90 & $-0,51$ \\
\hline LA 1777 & 0,89 & $-0,90$ & 6,82 & $-2,73$ & $-0,35$ & 11,17 & $-0,43$ \\
\hline LA 2329 & 3,49 & 0,58 & $-4,40$ & 4,59 & 0,26 & $-13,58$ & 0,64 \\
\hline PI 126445 & 4,52 & 0,50 & 0,57 & $-2,22$ & $-0,18$ & 1,51 & $-0,31$ \\
\hline PI 127826 & $-2,09$ & 0,49 & $-6,07$ & 0,43 & 0,17 & 6,05 & 0,03 \\
\hline PI 134417 & $-2,43$ & 1,69 & $-5,26$ & 3,42 & 0,32 & $-13,05$ & 0,57 \\
\hline
\end{tabular}

${ }^{1}$ MNP: Número de minas pequenas; MNG: Número de minas grandes; MLV: Mortalidade larval; PES: Peso pupal; PLV: Período larval; MPU: Mortalidade pupal; PPU: Período pupal.

das. Neste contexto, os sistemas de cruzamentos dialélicos são importante alternativa de avaliação dos genótipos, pois além de indicar os melhores híbridos, auxiliam na escolha dos genitores mais promissores a serem utilizados nos programas de hibridação (Cruz e Regazzi, 1994).

Os objetivos deste trabalho foram avaliar o potencial genético e a capacidade de combinação entre grupos de genótipos de Lycopersicon spp. quanto à resistência a Tuta absoluta.

\section{MATERIAL E MÉTODOS}

Este experimento foi conduzido em casas de vegetação da Universidade Federal de Viçosa, entre os meses de março e dezembro de 2000. A parcela experimental foi constituída por uma planta tomateiro com dois meses de idade, no delineamento experimental em blocos casualizados com quatro repetições. Os insetos utilizados foram provenientes da criação massal em laboratório da UFV, conforme metodologia desenvolvida por Miranda et al. (1998).

Foram obtidos 24 híbridos provenientes dos cruzamentos em esquema dialélico parcial, entre genitores do grupo I: Ângela, IPA-5, Santa Clara e TX 401-08 de Lycopersicon esculentum (padrões de suscetibilidade a T. absoluta, conforme observado por Leite et al., 1995; Picanço et al., 1995; Suinaga et al., 1999) e parentais pertencentes ao grupo II (portadores de resistência a $T$. absoluta): Lycopersicon hirsutum $\mathrm{f}$. typicum (LA 1777, LA 2329 e PI 126445); L. hirsutum f. glabratum (PI 134417), L. hirsutum f. hirsutum (PI 127826); e L. pennellii (LA 716). Nestas plantas foram inoculados 10 ovos de T. absoluta nos folíolos medianos do terço médio do limbo, sendo que, a partir da eclosão, as lagartas de T. absoluta foram confinadas em gaiolas de organza de $20 \times 28 \mathrm{~cm}$. Os caracteres avaliados neste experimento foram: a) Número de minas pequenas (MNP), amostrado pela contagem de minas com comprimento inferior a 0,5 centímetros; b) Número de minas grandes (MNG), incluindo minas com comprimento superior a 0,5 centímetros; c) Mortalidade larval (MLV), obtendo a percentagem da mortalidade de lagartas de $T$. absoluta; d) Período larval (PLV), obtido pela contagem de dias desde a eclosão da lagarta de T. absoluta até formação da pupa; e) Peso de pupas (PES) em mg de pupas de T. absoluta; f) Mortalidade pupal (MPU), obtida pela percentagem da mortalidade de pupas de T. absoluta; $\mathrm{g}$ ) Período pupal (PPU), compreendendo o período (dias) entre o primeiro estágio pupal e a emergência do adulto de T. absoluta.

Utilizando-se a metodologia adaptada de Suinaga et al. $(1999,2003)$, considerou-se resistente os genótipos que demonstraram características significativamente $(p<0,05)$ diferentes dos padrões de suscetibilidade. Assim, os genótipos que possuíam maiores estimativas do número de minas pequenas, mortalidade e período larvais, bem como a mortalidade e períodos pupais eram considerados resistentes. Por outro lado, os genótipos que promoviam a diminuição significativa $(p<0,05)$ do número de minas grandes e do peso de pupas, contribuíam para o aumento da expressão da resistência à traça do tomateiro.

A análise dialélica parcial foi realizada de acordo com o Método 2 (pais e $\mathrm{F}_{1 \mathrm{~s}}$ ), Modelo I (efeito de genótipo fixo), de Griffing (1956) adaptado a dialelos parciais por Geraldi e Miranda Filho (1988). As análises estatísticas foram realizadas com o auxílio do programa computacional GENES (Cruz, 2001).

\section{RESULTADOS E DISCUSSÃO}

As estimativas dos efeitos da capacidade geral de combinação $\left[\mathrm{CGC}\left(\mathrm{g}_{\mathrm{i}}\right)\right]$ dos parentais pertencentes aos grupos I e II, bem como as estimativas da capacidade específica de combinação $\left[\mathrm{CEC}\left(\mathrm{s}_{\mathrm{ii}}\right.\right.$ e $\left.\mathrm{s}_{\mathrm{ij}}\right)$ estão apresentados nas Tabelas 1 e 2 .

Na cultivar Santa Clara do grupo I foram constatados os menores valores $\mathrm{g}_{\mathrm{i}}$ de MNP(-2,93) e MNG $(-1,49)$. Por outro lado, no genitor Ângela, observa- 
Tabela 2. Estimativas dos efeitos da capacidade específica de combinação $\left(\mathrm{s}_{\mathrm{ii}}\right.$ and $\mathrm{s}_{\mathrm{ij}}$ ) para sete caracteres de resistência de tomateiros a $T$. absoluta. Viçosa, UFV, 2001.

\begin{tabular}{|c|c|c|c|c|c|c|c|}
\hline \multirow{2}{*}{ Efeitos $s_{i i}$} & \multicolumn{7}{|c|}{ Caracteres $^{1}$} \\
\hline & MNP & MNG & MLV & PLV & PES & MPU & PPU \\
\hline I & $-7,84$ & 1,77 & $-4,97$ & $-8,99$ & $-0,86$ & 12,00 & $-2,11$ \\
\hline II & $-12,51$ & 0,99 & $-19,04$ & $-1,02$ & $-0,12$ & 16,40 & $-0,23$ \\
\hline III & $-0,49$ & $-2,20$ & $-24,31$ & $-3,43$ & 0,94 & 18,70 & $-0,11$ \\
\hline IV & $-8,42$ & $-5,45$ & $-10,61$ & $-7,42$ & $-0,43$ & 26,63 & $-2,74$ \\
\hline 1 & $-2,36$ & 1,15 & $-10,65$ & $-10,45$ & $-1,09$ & 31,20 & $-2,33$ \\
\hline 2 & $-6,90$ & $-1,77$ & $-7,63$ & $-11,97$ & $-0,82$ & 24,66 & $-2,48$ \\
\hline 3 & $-4,11$ & $-0,97$ & $-5,20$ & $-1,48$ & 0,36 & $-13,34$ & $-1,11$ \\
\hline 4 & $-6,18$ & $-3,81$ & $-0,13$ & $-4,99$ & $-0,23$ & 18,97 & $-0,23$ \\
\hline 5 & $-0,45$ & 1,46 & $-6,84$ & $-0,99$ & $-0,14$ & 14,16 & $-0,41$ \\
\hline 6 & $-9,26$ & $-0,95$ & $-28,47$ & 9,00 & 1,45 & $-1,90$ & 1,37 \\
\hline Efeitos sij & MNP & MNG & MLV & PLV & PES & MPU & PPU \\
\hline $\mid x 1$ & $-0,10$ & $-2,54$ & 9,02 & 9,20 & 1,05 & $-28,40$ & 1,91 \\
\hline $1 \times 2$ & 8,38 & $-0,25$ & 13,70 & 6,02 & 0,25 & $-23,42$ & 1,46 \\
\hline $1 \times 3$ & 6,19 & $-0,73$ & 4,91 & $-2,72$ & 0,02 & 22,08 & $-0,27$ \\
\hline $1 \times 4$ & 4,16 & $-0,56$ & $-10,05$ & 6,66 & 0,35 & $-0,26$ & 1,15 \\
\hline $1 \times 5$ & $-5,64$ & 0,37 & $-18,41$ & 1,30 & 0,34 & 13,45 & $-0,54$ \\
\hline $1 \times 6$ & 2,70 & 0,16 & 10,78 & $-2,46$ & $-0,29$ & $-7,45$ & 0,50 \\
\hline II x 1 & $-1,23$ & $-0,10$ & 8,49 & 0,35 & 0,08 & $-1,20$ & $-0,17$ \\
\hline II $\times 2$ & $-5,16$ & 0,20 & $-9,59$ & 9,01 & 1,21 & $-23,22$ & 1,63 \\
\hline $11 \times 3$ & $-1,77$ & 0,22 & $-10,37$ & 2,06 & $-0,06$ & $-0,47$ & 1,44 \\
\hline II x 4 & 16,95 & 3,05 & 17,91 & $-5,26$ & $-0,52$ & $-11,56$ & $-1,62$ \\
\hline II $\times 5$ & 6,31 & $-4,69$ & 7,89 & 7,43 & 0,69 & 0,65 & 1,79 \\
\hline II x 6 & 9,91 & $-0,65$ & 23,75 & $-11,56$ & $-1,16$ & 3,00 & $-2,62$ \\
\hline III x 1 & $-4,93$ & $-3,02$ & 17,77 & 4,32 & 0,07 & $-25,05$ & 1,63 \\
\hline III x 2 & 8,14 & 1,94 & 15,28 & 3,81 & $-0,56$ & 9,18 & $-0,19$ \\
\hline III x 3 & $-1,05$ & $-0,96$ & 3,75 & 1,74 & $-0,06$ & 4,68 & $-0,05$ \\
\hline III x 4 & $-9,83$ & 3,62 & $-34,72$ & 8,67 & 1,14 & $-11,41$ & 1,43 \\
\hline III x 5 & 0,78 & 1,38 & 30,43 & $-8,59$ & $-1,33$ & $-23,20$ & $-1,78$ \\
\hline III x 6 & 7,88 & 1,43 & 16,11 & $-3,09$ & $-1,15$ & 8,40 & $-0,82$ \\
\hline IV $\times 1$ & 10,98 & 3,35 & $-13,96$ & 7,02 & 0,99 & $-7,75$ & 1,27 \\
\hline $\mathrm{IV} \times 2$ & 2,46 & 1,64 & $-4,12$ & 5,10 & 0,73 & $-11,85$ & 2,07 \\
\hline IV $\times 3$ & 4,86 & 3,42 & 12,10 & 1,86 & $-0,61$ & 0,40 & 1,11 \\
\hline IV $\times 4$ & 1,08 & 1,50 & 27,13 & $-0,08$ & $-0,52$ & $-14,70$ & $-0,51$ \\
\hline IV $\times 5$ & $-0,56$ & 0,01 & $-6,22$ & 1,84 & 0,58 & $-19,23$ & 1,35 \\
\hline IV $\times 6$ & $-1,97$ & 0,97 & 6,30 & $-0,89$ & $-0,30$ & $-0,14$ & 0,19 \\
\hline
\end{tabular}

${ }^{1}$ MNP: Número de minas pequenas; MNG: Número de minas grandes; MLV: Mortalidade larval; PES: Peso pupal; PLV: Período larval; MPU: Mortalidade pupal; PPU: Período pupal.

${ }^{2}$ I: Ângela; II: IPA-5; III: Santa Clara; IV: TX- 401-08

1: LA 716, 2: LA 1777, 3: LA 2329; 4: PI 126445, 5: PI 127826, e 6: PPI 134417

ram-se os maiores valores de CGC nas características PLV $(2,42)$ e MPU $(3,22)$. Considerando os genótipos pertencentes ao grupo II e o caráter MNP, os genitores com menor contribuição foram LA 716
$(-4,38)$ e PI $134417(-2,43)$. O genitor que influenciou negativamente o número de minas grandes foi LA $716(-2,36)$. Fato similar foi observado nos acessos LA $1777(-0,35)$ e LA $716(-0,21)$, com rela- ção ao caráter PES. Observou-se nestes mesmos acessos um aumento da mortalidade larval e pupal (Tabela 1).

Valores mais elevados da CGC indicam a importância dos genes predo- 
Tabela 3. Estimativas das médias (m) e heterose percentual (h) de sete caracteres de resistência de 24 híbridos de tomateiro a T. absoluta. Viçosa, UFV, 2001.

\begin{tabular}{|c|c|c|c|c|c|c|c|c|c|c|c|c|c|c|}
\hline \multirow{3}{*}{ Híbridos $^{2}$} & \multicolumn{14}{|c|}{ Caracteres ${ }^{1}$} \\
\hline & \multicolumn{2}{|c|}{ MNP } & \multicolumn{2}{|c|}{ MNG } & \multicolumn{2}{|c|}{ MLV } & \multicolumn{2}{|c|}{ PLV } & \multicolumn{2}{|c|}{ PES } & \multicolumn{2}{|c|}{ MPU } & \multicolumn{2}{|c|}{ PPU } \\
\hline & $m$ & $h$ & $\mathbf{m}$ & h & $m$ & $h$ & $m$ & $\mathrm{~h}$ & $m$ & h & $m$ & $h$ & $m$ & $h$ \\
\hline $1 \times 1$ & 8,5 & 142,9 & 4,5 & $-47,1$ & 66,8 & 33,7 & 32,9 & 135,1 & 3,5 & 133,0 & 0,0 & $-100,0$ & 7,8 & 113,6 \\
\hline $1 \times 2$ & 22,3 & 242,3 & 8,3 & $-2,9$ & 70,0 & 40,0 & 30,5 & 117,8 & 2,6 & 71,1 & 8,3 & $-83,5$ & 7,4 & 103,2 \\
\hline $1 \times 3$ & 22,7 & 115,9 & 9,3 & $-10,8$ & 50,0 & 25,0 & 29,1 & 9,5 & 3,0 & 9,8 & 29,0 & 364,0 & 6,7 & 24,9 \\
\hline $1 \times 4$ & 21,7 & 106,4 & 9,3 & 5,2 & 40,0 & $-15,8$ & 31,7 & 75,8 & 2,9 & 44,9 & 21,8 & $-42,0$ & 7,2 & 47,6 \\
\hline $1 \times 5$ & 5,3 & $-22,2$ & 10,3 & $-10,9$ & 25,0 & $-33,3$ & 28,9 & 27,8 & 3,2 & 35,2 & 40,0 & 0,9 & 5,8 & 13,9 \\
\hline $1 \times 6$ & 13,3 & 562,5 & 11,3 & $-2,2$ & 55,0 & 100,0 & 28,2 & $-8,1$ & 2,7 & $-17,4$ & 0,0 & $-99,9$ & 7,4 & 13,3 \\
\hline$\| \times 1$ & 9,7 & 179,5 & 7,0 & $-14,3$ & 73,3 & 46,7 & 19,8 & 44,2 & 2,2 & 45,0 & 25,0 & $-50,0$ & 4,8 & 30,2 \\
\hline$\| \times 2$ & 11,0 & 70,3 & 8,8 & 7,1 & 53,8 & 7,5 & 29,3 & 112,7 & 3,2 & 109,6 & 6,3 & $-87,5$ & 6,6 & 81,6 \\
\hline$\| \times 3$ & 17,0 & 62,5 & 10,3 & 2,1 & 41,8 & 4,4 & 29,6 & 12,6 & 2,6 & $-6,5$ & 4,3 & $-32,1$ & 7,5 & 39,2 \\
\hline II $\times 4$ & 36,8 & 251,4 & 13,0 & 52,2 & 75,0 & 57,9 & 15,5 & $-12,7$ & 1,6 & $-17,4$ & 8,3 & $-78,0$ & 3,5 & $-28,4$ \\
\hline$\| \times 5$ & 19,5 & 190,7 & 5,3 & $-53,0$ & 58,3 & 55,6 & 30,8 & 37,7 & 3,2 & 34,1 & 25,0 & $-36,9$ & 7,3 & 41,1 \\
\hline$\| \times 6$ & 22,8 & 10,61 & 10,5 & $-6,0$ & 75,0 & 172,7 & 14,8 & $-51,2$ & 1,5 & $-54,7$ & 8,3 & $-34,0$ & 3,4 & $-48,6$ \\
\hline III x 1 & 0,0 & $-99,4$ & 1,3 & $-66,7$ & 85,3 & 70,5 & 23,0 & 95,9 & 2,4 & 6,3 & 0,0 & $-100,0$ & 6,5 & 77,8 \\
\hline III x 2 & 18,3 & 182,0 & 7,7 & 104,4 & 81,3 & 62,5 & 23,3 & 98,0 & 1,7 & $-27,2$ & 37,5 & $-25,0$ & 4,8 & 30,0 \\
\hline III x 3 & 11,8 & 11,9 & 6,3 & 11,1 & 58,5 & 46,2 & 28,5 & 17,3 & 2,8 & $-20,2$ & 8,3 & 32,0 & 6,0 & 10,4 \\
\hline III $x 4$ & 4,0 & $-61,9$ & 10,8 & 160,6 & 25,0 & $-47,4$ & 28,6 & 81,9 & 3,5 & 28,3 & 7,3 & $-80,7$ & 6,5 & 32,8 \\
\hline III x 5 & 8,0 & 18,5 & 8,5 & 25,9 & 83,5 & 122,7 & 14,0 & $-31,3$ & 1,4 & $-55,1$ & 0,0 & $-100,0$ & 3,6 & $-29,5$ \\
\hline III x 6 & 14,8 & 637,5 & 9,8 & 44,4 & 70,0 & 154,5 & 22,5 & $-20,7$ & 1,8 & $-57,2$ & 12,5 & 0,0 & 5,1 & $-22,0$ \\
\hline IV $x 1$ & 18,0 & 1004,3 & 8,0 & 219,9 & 46,7 & $-6,7$ & 30,3 & 111,0 & 3,4 & 103,7 & 13,3 & $-73,3$ & 7,3 & 107,4 \\
\hline $\mathrm{IV} \times 2$ & 14,8 & 218,6 & 7,8 & 209,9 & 55,0 & 10,0 & 29,2 & 102,9 & 3,0 & 80,1 & 12,5 & $-75,0$ & 8,2 & 132,1 \\
\hline IV $\times 3$ & 19,8 & 128,9 & 11,0 & 151,4 & 60,0 & 50,0 & 33,3 & 23,4 & 2,3 & $-19,9$ & 0,0 & $-100,0$ & 8,3 & 57,5 \\
\hline IV $\times 4$ & 17,0 & 97,0 & 9,0 & 213,0 & 80,0 & 68,4 & 24,5 & 33,3 & 2,0 & $-8,7$ & 0,0 & $-100,0$ & 5,8 & 20,4 \\
\hline IV $\times 5$ & 8,8 & 79,3 & 7,5 & 36,4 & 40,0 & 6,7 & 29,1 & 26,2 & 3,4 & 33,8 & 0,0 & $-100,0$ & 8,0 & 58,2 \\
\hline IV $\times 6$ & 7,0 & 52,84 & 9,7 & 75,8 & 53,3 & 93,9 & 29,3 & $-5,4$ & 2,7 & $-23,2$ & 0,0 & $-94,4$ & 7,3 & 13,6 \\
\hline
\end{tabular}

${ }^{1}$ MNP: Número de minas pequenas; MNG: Número de minas grandes; MLV: Mortalidade larval; PES: Peso pupal; PLV: Período larval; MPU: Mortalidade pupal; PPU: Período pupal.

minantemente aditivos em seus efeitos (Hallauer e Miranda Filho, 1988). Desta forma, os genitores com maiores valores de CGC poderão estar presentes em programas de melhoramento genético cujo objetivo seja a seleção de linhagens derivadas de populações segregantes a partir de cruzamentos com os parentais testados (Carvalho et al., 1999). Considerando as magnitudes e os sinais das estimativas de $\mathrm{g}_{\mathrm{i}}$, os genótipos pertencentes ao grupo I e as características MNP, MNG, PLV, e MPU, as cultivares Santa Clara e Ângela são os genitores mais indicados a serem incluídos em programas de melhoramento do tomateiro que visem aumentar a resistência a T. absoluta. Com referência aos genitores do grupo II e as características MNP, MNG, MLV, PLV, PES, e PPU, os acessos LA 716 e LA 1777 são os mais recomendados a programas de melhoramento objetivando resistência a
T. absoluta. Estes genótipos foram escolhidos pois, apresentaram contribuições negativas, ou seja atuaram na diminuição do número de minas pequenas e grandes, peso de pupas da traça do tomateiro e, por outro lado, aumentaram a expressão da mortalidade e período larvais, bem como a mortalidade de pupas deste inseto. A manifestação da resistência à traça-do-tomateiro, presente nestes parentais, pode ser explicada pela ação de antibiose proporcionada pelos acilaçúcares presentes em LA 716 (Hawthorne et al., 1992; Goffreda et al, 1989; Campos et al., 1999) e sesquiterpenos atuantes no acesso LA 1777 (Ecole et al., 1999).

Quanto às estimativas de CEC $\left(\mathrm{s}_{\mathrm{ii}}\right)$, estas foram negativas em todos os genitores quanto às características MNP e MLV. A cultivar Santa Clara $(-0,49)$ e PI $127826(-0,45)$ apresentaram os maiores valores de MNP e Santa Clara
$(-24,31)$ e PI $134417(-28,47)$ as menores estimativas de MLV. Quanto aos caracteres PLV, PES e PPU tais valores também foram negativos com exceção do acesso PI 134417 (9,00; 1,45 e 1,37 respectivamente). Observou-se no acesso silvestre LA 2329 o menor valor de CEC da característica MPU $(13,34)$.

Com relação a MNP, nota-se que a combinação híbrida Santa Clara x PI $126445(-9,83)$ revelou o menor valor de CEC $\left(\mathrm{s}_{\mathrm{ij}}\right)$, enquanto que no caráter PES foram observadas menores estimativas de CEC nos híbridos Santa Clara $\mathrm{x}$ PI $127826(-1,33)$ e Santa Clara x PI $134417(-1,15)$. Contrariamente os cruzamentos que contribuíram positivamente com o aumento das características MLV, MPU, e PPU foram respectivamente Santa Clara x PI $127826(30,43)$, Ângela x LA 2329 (22,08), e Santa Clara x LA 1777 (2,07) (Tabela 2). 
O híbrido com os maiores valores negativos de heterose nas características MNP e MNG, foi Santa Clara x LA 716 (-99,4 e $-66,7$ respectivamente). Quanto à característica PES, a combinação híbrida na qual foi observado o menor valor heterótico foi Santa Clara x PI 134417 (-57,2). Por outro lado, os cruzamentos que expuseram os maiores valores de heterose na característica MLV foram IPA-5 x PI 134417 (172,7) e Santa Clara x PI $134417(154,5)$. Os maiores valores de heterose, considerando o caráter PLV, foram obtidas por Ângela x LA $716(135,1)$ e Ângela x LA $1777(117,8)$. Nas características MPU e PPU, os cruzamentos com as maiores magnitudes de heterose foram Ângela $\mathrm{X}$ LA 2329 (364,0) e TX 401-08 x LA 1777 $(132,1)$, respectivamente (Tabela 3$)$.

A respeito das estimativas de CEC, Cruz e Vencovsky (1989) relataram que estes valores, nos modelos aditivo-dominantes, correspondem em medida de divergência genética entre o genitor i e os demais presentes no dialelo. Assim, quanto maior o valor absoluto de $\mathrm{s}_{\mathrm{ii}}$, maior será a divergência genética do genitor i em relação aos demais. Ainda segundo Cruz e Vencovsky (1989), outro fator importante a ser analisado é o sinal das estimativas de $\mathrm{s}_{\mathrm{ii}}$, pois quando este é negativo, o genitor i contribuirá positivamente com a heterose; no caso contrário negativamente e quando for zero ou próximo deste, a divergência genética deste genitor com os demais será pequena ou nula e a heterose das combinações híbridas entre o genitor i tende a ser pequena. Desta forma, os genitores Ângela e Santa Clara (grupo I) e os acessos LA 716, LA 1777 e PI 134417 (grupo II) podem apresentar as maiores divergências genéticas, manifestar positiva ou negativamente heterose, e contribuir na elevação das características relativas a mortalidade e ciclo de vida da traça do tomateiro, bem como, diminuir aquelas referentes ao número de minas e peso de pupas de T. absoluta.

Segundo Griffing (1956), as melhores combinações devem possuir altos valores de $\mathrm{s}_{\mathrm{ij}}$ e altas estimativas de CGC. Além destes quesitos, a avaliação da heterose presente nestes híbridos pode auxiliar na escolha das melhores combinações (Amaral Júnior et al., 1996). Assim, tendo por base as estimativas de capacidade geral e específica, e o valor heterótico destes híbridos as combinações Ângela x PI 126445, Ângela x PI 127826, IPA-5 x LA 1777, e Santa Clara x LA 716 têm alto potencial no melhoramento genético do tomateiro visando resistência a $T$. absoluta, pois apresentaram pelo menos um genitor com altas CGC e CEC, e também expressivo valor heterótico.

\section{LITERATURA CITADA}

AMARAL JÚNIOR, A.T.; CASALI, V.W.D.; CRUZ, C.D.; FINGER, F.L. Utilização das variáveis canônicas e de análise de agrupamentos na avaliação da divergência genética entre acesso de moranga. Horticultura Brasileira, Brasília, v.14, n.2, p.182-184, 1996.

CAMPOS, G.A., MALUF; W.R., CARDOSO, M.G.; BRAGA, L.R.; TEODORO, A.V.; GUIMARÃES, E.R.; BENITES, F.R.G.; AZEVEDO, S.M.; RESENDE, J.T.V. Resistência de tomateiros com altos teores de zingibereno, ou 2tridecanona oriundos de cruzamentos interespecíficos de Lycopersicon a ácaros do gênero Tetranychus. Horticultura Brasileira, Brasília, v.17, n.3, p.33, 1999.

CARVALHO, A.C.P.P.; LEAL, N.R.; RODRIGUES, R.; COSTA, F.A. Capacidade de combinação para oito caracteres agronômicos em cultivares de feijão-de-vagem de crescimento determinado. Horticultura Brasileira, Brasília, v. 17, n.2, p. 102-105, 1999.

CRUZ, C.D. Programa GENES: aplicativo computacional em genética e estatística. Viçosa: Imprensa Universitária, 2001. 442 p.

CRUZ, C.D.; REGAZZI, A.J. Modelos biométricos aplicados ao melhoramento genético. Viçosa: Imprensa Universitária, 1994. 390 p. CRUZ, C.D.; VENCOVSKY, R. Comparação de alguns métodos de análise dialélica. Revista Brasileira de Genética, Ribeirão Preto, v.12, n.2, p.425-438, 1989.

DeVERNA, J.W.; PATERSON, A.H. Genetics of Lycopersicon. In: KALLOO, G. (Ed.). Genetic improvement of tomato. Berlin: Springer Verlag, 1991. p. 21-38.

ECOLE, C.C.; PICANÇO, M.; JHAM, G.N.; GUEDES, R.N.C. Variability of Lycopersicon hirsutum f. typicum and possible compounds involved in its resistance to Tuta absoluta. Agricultural and Forest Entomology, v.1, p.249254, 1999.

EIGENBRODE, S.D.; TRUMBLE, J.T. Antibiosis to beet armyworm (Spodoptera exigua) in Lycopersicon accessions. HortScience, v.28, n.5, p.932-934, 1993.

EIGENBRODE, S.D.; TRUMBLE, J.T. Fruit based tolerance to damage by beet armyworm (Lepidoptera: Noctuidae) in tomato. Environmental Entomology, v.23, n.4, p.937-942, 1994.

EIGENBRODE, S.D.; TRUMBLE, J.T.; MILLAR, J.G.; WHITE, K.K. Topical toxicity of tomato sesquiterpenes to the beet armyworm and the role of these compounds in resistance derived from an accession of Lycopersicon hirsutum $\mathrm{f}$. typicum. Journal of Agricultural Food Chemistry, v.42, n.3, p.807-810, 1994.
EIGENBRODE, S.D.; TRUMBLE, J.T.; WHITE, K.K Trichome exudates and resistance to beet armyworm (Lepidoptera: Noctuidae) in Lycopersicon hirsutum f. typicum accessions. Environmental Entomology, v.25, n.1, p.90-95, 1996.

FARRAR JR, R.R.; KENNEDY, G.G. Relationship of leaf lamellar-based resistance to Leptinotarsa decemlineata and Heliothis zea in a wild tomato, Lycopersicon hirsutum f. glabratum, PI 134417. Entomologia Experimentalis et Applicata, v.58, n.1, p.61-67, 1991.

FARRAR JR, R.R.; KENNEDY, G.G. 2undecanone, a constituent of the glandular trichomes of Lycopersicon hirsutum f glabratum effects on Heliothis zea and Manduca sexta growth and survival. Entomologia Experimentalis et Applicata, v.43, n.1, p.17-23, 1987.

GERALDI, I.O.; MIRANDA FILHO, J.B. Adapted models for the analysis of combining ability of varieties in partial diallel crosses. Revista Brasileira de Genética, Ribeirão Preto, v.11, n.2, p.419-430, 1988.

GOFFREDA, J.C.; MUTSCHLER, M.A.; AVE, D.A.; TINGEY, W.M.; STEFFENS. Aphid deterrence by glucose esters in glandular trichome exudate of the wild tomato, Lycopersicon pennellii. Journal of Chemical Ecology, v.15, p.2135-2147, 1989.

GRIFFING, B. Concept of general and specific combining ability in relation to diallel crossing systems. Australian Journal of Biological Sciences, v.9, n.3, p.463-493, 1956.

GUEDES, R.N.C.; PICANÇO, M.C.; GUEDES, N.M.P.; MADEIRA, N.R. Sinergismo do óleo mineral sobre a toxicidade de inseticidas para Scrobipalpuloides absoluta (Lepidoptera: Gelechiidae). Pesquisa Agropecuária Brasileira, Brasília, v.30, n.4, p.313-318, 1995.

HALLAUER, S.R.; MIRANDA FILHO, J.B. Quantitative genetics in maize breeding. 2ed. Ames: Iowa State University Press, 1988. 468 p. HAWTHORNE, D.J.; SHAPIRO, J.A; TINGEY, W.M.; MUTSCHLER, M.A. Trichome-borne and artificially applied acylsugars of wild tomato deter feeding and oviposition of the leafminer Liriomyza trifolii. Entomologia Experimentalis et Applicata, v.65, n.1, p.65-73, 1992.

LEITE, G.L.D., PICANÇO, M., SILVA, D.J.H. Distribuição de oviposição de Scrobipalpuloides absoluta no dossel de Lycopersicon esculentum, L. hirsutum e L. peruvianum. Horticultura Brasileira, Brasília, v.13, n.1, p.47-51, 1995.

LEITE, G.L.D.; PICANÇO, M.; GUEDES, R.N.C.; ZANUNCIO, J.C. Influence of canopy height and fertilization levels on the resistance of Lycopersicon hirsutum to Aculops lycopersici. Experimental and Applied Acarology, v.23, n.2, p.633-642, 1999a.

LEITE, G.L.D.; PICANÇO, M.; GUEDES, R.N.C.; SKOWRONSKI, L. Effect of fertilization levels, age and canopy height of Lycopersicon hirsutum on resistance to Myzus persicae. Entomologia Experimentalis et Applicata, v.91, n.2, p.267-273, 1999b.

LIN, S.Y.H.; TRUMBLE, J.T. Resistance in wild tomatoes to larvae of a specialist herbivore Keiferia lycopersicella. Entomologia Experimentalis et Applicata, v.41, n.1, p.53-60, 1986. 
MELO, M.; CAMPOS, A.D. Ocorrência de inimigos naturais da traça-do-tomateiro Tuta absoluta (Meyrick, 1917) (Lepidoptera: Gelechiidae) em Pelotas, Rio Grande do Sul. Agropecuária de Clima Temperado, Passo Fundo, v.3, n.2, p.269274, 2000.

MIRANDA, M.M.M.; PICANCO, M.C. ZANUNCIO, J.C.; GUEDES, R.N.C. Ecologica life table of Tuta absoluta (Meyrick) (Lepidoptera: Gelechiidae). Biocontrol Science and Technology, v.8, n.4, p.597-606, 1998.

MOREIRA, L.F.; OLIVEIRA, J.S. Análise de resíduos de metamidofós em frutos de tomate, água e solo da região agrícola de Viçosa-MG. Revista Ceres, Viçosa, v.44, n.252, p.161-168, 1997.

PEDIGO, L.P. Entomology and pest management. New Jersey: Prentice Hall, 2002. 742 p.

PICANÇO, M.C., SILVA, D.J.H., LEITE, G.L.D. Intensidade de ataque de Scrobipalpula absoluta (Meyrick, 1917) ao dossel de três espécies de tomateiro. Pesquisa Agropecuária Brasileira, Brasília, v.30, n.2, p.429-433, 1995.
PICANÇO, M.; FALEIRO, F.G.; PALLINI FILHO, A.; MATIOLI, A.L. Perdas na produtividade do tomateiro em sistemas alternativos de controle fitossanitário. Horticultura Brasileira, Brasília, v.15, n.2, p.88-91, 1997.

RAMALHO, M.A.P.; SANTOS, J.B.; ZIMMERMAN, M.J.O. Genética quantitativa aplicada em plantas autógamas: aplicação ao melhoramento do feijoeiro. Goiânia: UFG, 1993. 271 p. SIQUEIRA, H.A.A.; GUEDES, R.N.C.; PICANÇO, M.C. Insecticide resistance in populations of Tuta absoluta (Lepidoptera: Gelechiidae). Agricultural and Forest Entomology, v.2, n.2, p.147-153, 2000.

SIQUEIRA, H.A.A.; GUEDES, R.N.C.; FRAGOSO, D.B.; MAGALHÃES, L.C. Abamectin resistance and synergism in Brazilian populations of Tuta absoluta (Meyrick) (Lepidoptera: Gelechiidae). International Journal of Pest Management, v.47, n.2, p.247-251, 2001.
SUINAGA, F.A.; PICANÇO, M.; JHAN, G.N.; BROMMONSCHENKEL, S.H. Causas químicas da resistência de Lycopersicon peruvianum a Tuta absoluta. Anais da Sociedade Entomológica do Brasil, v.28, n.2, p.313-321, 1999.

SUINAGA, F.A.; CASALI, V.W.D.; SILVA, D.J.H.; PICANÇO, M. Dissimilaridade genética de fontes de resistência de Lycopersicon spp. a Tuta absoluta (Lepidoptera: Gelechiidae). Revista Brasileira de Agrociência, v.9, n.4, p.371-376, 2003.

TOSCANO, L.C.; BOIÇA Jr., A.L. Atratividade de genótipos de tomateiro Lycopersicon spp. a mosca branca Bemisia argentifolii. Horticultura Brasileira, Brasília, v.17, n.3, p.33, 1999. 